\title{
Influence of type I IFN signaling on anti- MOG antibody-mediated demyelination
}

\author{
Carsten Tue Berg ${ }^{1}$, Reza Khorooshi ${ }^{1}$, Nasrin Asgari ${ }^{1,2}$ and Trevor Owens ${ }^{1 *}$ (D)
}

\begin{abstract}
Background: Antibodies with specificity for myelin oligodendrocyte glycoprotein (MOG) are implicated in multiple sclerosis and related diseases. The pathogenic importance of anti-MOG antibody in primary demyelinating pathology remains poorly characterized.

Objective: The objective of this study is to investigate whether administration of anti-MOG antibody would be sufficient for demyelination and to determine if type I interferon (IFN) signaling plays a similar role in anti-MOG antibody-mediated pathology, as has been shown for neuromyelitis optica-like pathology.

Methods: Purified IgG2a monoclonal anti-MOG antibody and mouse complement were stereotactically injected into the corpus callosum of wild-type and type I IFN receptor deficient mice (IFNAR1-KO) with and without preestablished experimental autoimmune encephalomyelitis (EAE).

Results: Anti-MOG induced complement-dependent demyelination in the corpus callosum of wild-type mice and did not occur in mice that received control lgG2a. Deposition of activated complement coincided with demyelination, and this was significantly reduced in IFNAR1-KO mice. Co-injection of anti-MOG and complement at onset of symptoms of EAE induced similar levels of callosal demyelination in wild-type and IFNAR1-KO mice.

Conclusions: Anti-MOG antibody and complement was sufficient to induce callosal demyelination, and pathology was dependent on type I IFN. Induction of EAE in IFNAR1-KO mice overcame the dependence on type I IFN for anti-MOG and complement-mediated demyelination.
\end{abstract}

Keywords: Beta-interferon, Demyelination, Histopathology, Immunology, Animal model, Experimental autoimmune encephalomyelitis, Multiple sclerosis

\section{Introduction}

Multiple Sclerosis (MS) is an inflammatory demyelinating disease of the central nervous system (CNS) with a complex pathogenesis [1]. MS pathology has been characterized by chronic inflammation, which leads to focal plaques of demyelination in the white matter [2]. More than $50 \%$ of MS lesions show complement $(C)$ and immunoglobulin G (IgG) deposition on demyelinating axons, classified as type II pathology [3, 4], but there is no consensus as to either the specificity or pathologic role of IgG antibodies in blood or cerebrospinal fluid (CSF) of MS patients [1]. Plasma exchange, which removes IgGs, as well as immune complexes and

\footnotetext{
* Correspondence: towens@health.sdu.dk

${ }^{1}$ Institute of Molecular Medicine, Neurobiology, University of Southern

Denmark, JB. Winsloewsvej 25, 5000 Odense C, Denmark

Full list of author information is available at the end of the article
}

cytokines, was effective for a group of patients confirmed to have had type II MS pathology [5]. This suggests that antibody and $\mathrm{C}$ are effectors of pathology in MS. Localization of $\mathrm{C}$ deposition has been shown in areas of active demyelination in patients with IgG myelin oligodendrocyte glycoprotein (MOG)-antibody-associated encephalomyelitis and pattern II MS [6-8]. One candidate autoantigen in MS is MOG, which is expressed by oligodendrocytes and on the outermost surface of the myelin sheath $[9,10]$. Autoantibodies against MOG are implicated in pediatric MS [11] and acute disseminated encephalomyelitis (ADEM) [12] as well as in a subset of water channel aquaporin-4 (AQP4)-IgG seronegative neuromyelitis optica spectrum disorder (NMOSD) [13-15].

The most extensively used animal model of MS, experimental autoimmune encephalomyelitis (EAE), is an adjuvant-driven experimental autoimmune disease, which 
in most formulations is dependent on CD4+ T cells. In a commonly used EAE variant, immunization of C57BL/6 mice with an encephalitogenic peptide (MOG p35-55) induces a chronic inflammatory demyelinating disease that has been shown to be independent of IgG or B cells [16], whereas immunization with recombinant human MOG or a fusion protein of myelin basic protein and proteolipid protein induces EAE that is dependent on B cells and antibody [17-20]. It has previously been shown that injection of monoclonal antibody against MOG into Lewis rats with EAE induced demyelination [21, 22]. Serum from guinea pigs with chronic-relapsing EAE could also induce demyelination, when transferred into subarachnoid space of normal rats and the degree of demyelination correlated with high titers of anti-MOG antibody in serum [22]. However, direct demonstration of demyelination induced by MOGspecific antibody has not been reported. We have previously demonstrated complement-dependent astrocytopathology and demyelination in mice that received neuromyelitis optica (NMO) patient-derived IgG [23]. We also showed a requirement for type I IFN signaling for NMO-like pathology, showing that it was reduced in mice lacking the receptor for type I IFN [24]. This observation aligned with clinical findings that recombinant IFN- $\beta$ is not an effective therapy for NMO whereas it shows effect for MS [25-27]. Interestingly, MOG-IgG-positive patients also showed increased disease activity under treatment with IFN- $\beta[14,28]$. The objective of this study was to extend those findings, to investigate whether IFNAR dependency would be seen for other antibody specificities. We chose to test a monoclonal MOG-specific antibody. This required establishing that anti-MOG antibody is sufficient to induce primary demyelinating pathology and then to ask whether and how type I IFN signaling affects that pathology in mice with and without EAE.

\section{Materials and methods \\ Mice}

Adult female C57BL/6 mice aged 8-12 weeks were purchased from Taconic Europe (Ry, Denmark), and adult female type I IFN receptor deficient mice (IFNAR1-KO) aged $8-12$ on C57BL/6 background were bred from mice originally provided by Dr. Marco Prinz. NOD-Scid/J immunodeficient mice aged 8-12 with impaired $\mathrm{T}$ and $\mathrm{B}$ cell lymphocyte development were obtained from Prof. Moustapha Kassem. All experiments were conducted in accordance with the Danish Animal Experiments Inspectorate (approval number 2014-15-0201-00369).

\section{EAE induction}

C57BL/6 and IFNAR1-KO mice were immunized with MOG p35-55 (sequence MEVGWYRSPFSRVVHLYRNGK), obtained from TAG Copenhagen A/S in Denmark.
Emulsions of MOG p35-55 (300 $\mu \mathrm{g})$ and complete Freund's adjuvant with heat-inactivated Mycobacterium tuberculosis (200 $\mu \mathrm{g}$; BD-Biosciences, Sparks, USA) were injected subcutaneously into each hind flank. Animals received an intraperitoneal injection of pertussis toxin (0.3 $\mu$ g; Sigma-Aldrich, Brøndby, Denmark) at the time of immunization and 2 days post-immunization. Mice were monitored for the loss of body weight and EAE symptoms as described previously [29].

\section{Intracorpus callosum injection}

Mice were anesthetized using hypnorm (fluanisone/ fentanyl) in combination with midazolam. Intracorpus callosum (CC) injections were performed using the following stereotactic coordinates relative to bregma: $1 \mathrm{~mm}$ anterior, $1 \mathrm{~mm}$ lateral, and -1.6 ventral. Anti-MOG IgG2a (15 $\mu \mathrm{g} /$ mouse) (protein $\mathrm{G}$ affinity purified supernatant from hybridoma clone $\mathrm{Z} 2$ developed by Prof. Chris Linington and IgG2a isotype control from murine myeloma (Sigma-Aldrich, Brøndby, Denmark) were used in the experiments. Mice received intra-CC injection $(5 \mu \mathrm{l})$ of anti-MOG $\mathrm{Ab}$ or control IgG2a together with $2 \mu$ mouse $\mathrm{C}$ (Sigma-Aldrich; Cedarlane, Burlington, Canada). The mice were euthanized 2 days post-injection with an overdose of pentobarbital ( $0.2 \mathrm{mg}$ per gram body weight, Glostrup Apotek, Glostrup, Denmark) and perfused transcardially with ice-cold phosphate buffered saline (PBS). For q-RT-PCR analysis, CNS tissue were dissected and immediately placed in TRIzol reagent and stored at $-80{ }^{\circ} \mathrm{C}$ until further q-RT-PCR processing. For histology, mice were additionally perfused with 4\% PFA in PBS. After removal, CNS tissue was post-fixed in $4 \%$ paraformaldehyde, immersed in $30 \%$ sucrose in $\mathrm{PBS}$ at $4{ }^{\circ} \mathrm{C}$ overnight, frozen with liquid nitrogen and stored at $-80{ }^{\circ} \mathrm{C}$ until sections were cut on a cryostat.

\section{RNA extraction, reverse transcription, and quantitative real-time PCR}

RNA was extracted from brain tissue using TRIzol reagent (Invitrogen-Molecular Probes, Eugene OR USA) in accordance with the manufacture's protocol. One microgram of total RNA was reverse transcribed using M-MLV reverse transcriptase (Invitrogen) according to the manufacturer's protocol. The following sequencespecific primers and probes were used for IFN- $\beta$ and CXCL10: IFN-ß: For: GCGTTCCTGCTGTGCTTCTC; Rev: TTGAAGTCCGCCCTGTAGGT; Probe: CGG AAATGTCAGGAGCT; CXCL10: For: GCCGT CA TTTTCTGCCTCAT; Rev: GGCCCGTCATCGATAT GG; Probe: GGACTCAAGGGATCC. All samples were run as triplicates and plates detecting the same gene 
were normalized using a generalized sample of cDNA. The relative quantitation of gene expression was determined using the delta cycle threshold $(\Delta \mathrm{Ct})$ method.

\section{Histochemistry and immunohistochemistry}

For routine histology, $12-\mu \mathrm{m}$ thick sections were cut from frozen brain and spinal cord tissue and mounted on Superfrost ${ }^{\circ}$ Plus (Thermo Scientific, Braunschweig, Germany) slides and stained using luxol fast blue (LFB) and hematoxylin and eosin (H\&E) staining. Immunohistochemical staining used to identify and examine $\mathrm{C}$ deposition, astrocyte and microglia activation were performed by sequential antibody incubations and detection. The primary antibody used to identify $\mathrm{C}$ deposition, activation of astrocytes, and microglia were C9neo (rabbit polyclonal anti-C5b-9, 1:100, Abcam, Cambridge, UK), GFAP (rabbit anti-mouse glial fibrillary acidic protein, 1:500, Dako, Glostrup, Denmark), and Iba-1 (rabbit anti-ionized calcium binding adapter molecule 1 (1:500, Waco, Osaka, Japan), respectively. The secondary antibodies were goat anti-rabbit conjugated with biotin (Abcam, 1:1, Cambridge, UK) followed by streptavidin-horseradish peroxidase (streptavidin-HRP, 1:200, Amersham Biosciences, Little Buckinghamshire, $\mathrm{UK})$ and $\mathrm{DAB}$ detection (3,3'-diaminobenzidine, $0.5 \mathrm{mg} / \mathrm{ml}$, Sigma Aldrich). For double staining for infiltration of CD45-positive cells and laminin, frozen brain sections were incubated with primary antibodies--rat anti-mouse CD45 conjugated with PE (BD Biosciences, San Diego, CA, USA (1:300)) and rabbit anti-mouse laminin (Cederlane 1:100)--and the corresponding fluorophore-conjugated secondary antibody--donkey anti-rabbit IgG conjugated with Alexa fluor 488 (Invitrogen 1:200). Cellular nuclei were visualized by the use of 4,6-diamino-2-phenylindol (DAPI, Invitrogen) diluted in PBS.

\section{Quantitation of histological and immunohistochemical findings}

For all analyses, brain and spinal cord sections were coded, analyzed, and quantified blindly. The area of demyelination in CC was quantitated using ImageJ software. The number of infiltrating cells in the $\mathrm{CC}$ was determined manually by counting the number of CD45 positive cells. The evaluation of the activation of astrocytes and microglia in and around demyelinated areas was performed blinded and semi-quantitatively using a scale from 0 to 3 as described [23].

\section{Data presentation and statistical analysis}

All experiments were repeated at least three times, and data are presented as mean \pm standard error of mean (SEM). Statistical significance was assessed by the twotailed Mann-Whitney $U$ test using GraphPad prism v. 6.0e software (GraphPad Software, USA). $P$ values $<0.05$ were considered as evidence of statistical significance.

\section{Results}

Callosal demyelination by anti-MOG and complement

To investigate whether a monoclonal MOG-specific antibody would be sufficient to induce MS-related pathology in the presence of $C$, we injected 5,15 , and $30 \mu \mathrm{g}$ anti-MOG into $\mathrm{CC}$ and examined pathology 2 days later. Focal pathology corresponding to antibody and Cmediated damage was observed at the site of injection, in the CC. Significant demyelination was detectable at anti-MOG $\geq 15 \mu \mathrm{g}$, and this dose was used in all subsequent experiments (Fig. 1a). Demyelination was dependent on co-injection of $\mathrm{C}$, and did not occur in mice that received anti-MOG alone, or control IgG2a with C (Fig. 1b, c). Deposition of activated C could be demonstrated at the same site as demyelination (Fig. 1b).

Some inflammation was seen, which was accounted for by the trauma of needle insertion.

A few CD45-positive cells were located mainly within the needle track. We also observed activation of astrocytes (GFAP staining) and microglia (Iba1 staining) in and around demyelinated areas (Fig. 2a).

Demyelination was equivalent in NOD-Scid/J immunodeficient mice to which anti-MOG $+\mathrm{C}$ was injected into the CC (Fig. 1c). This shows that anti-MOG $+\mathrm{C}$-mediated demyelination is independent of $\mathrm{B}$ and $\mathrm{T}$ cells.

\section{Induction of type I IFN and influence of type I IFN} signaling on anti-MOG antibody-mediated demyelination We used RT-qPCR to evaluate gene expression for IFNbeta (IFN- $\beta$ ), a type I IFN, and the IFN-induced chemokine CXCL10, in brain RNA isolates. We observed high expression of IFN- $\beta$ mRNA compared to controls (Fig. 2b). Additionally, we observed a significant increase in gene expression for CXCL10. This points to a potential role for type I IFN signaling in antibody-mediated demyelination. We further investigated whether injection of anti-MOG $+\mathrm{C}$ led to pathology in IFNAR1-KO mice. Figure 2c shows that the lack of the IFNAR-1 receptor led to significantly reduced demyelination in $\mathrm{CC}$, when compared to C57BL/6 mice.

\section{EAE and IFN- $\gamma$ overcome dependency on type I IFN for anti-MOG $+\mathrm{C}$ induced demyelination}

To test whether demyelination was similarly affected by type I IFN in mice with EAE, disease was induced by immunization with MOG peptide 35-55 and CFA. The incidence of EAE was almost two times higher in IFNAR1$\mathrm{KO}$ mice compared to $\mathrm{C} 57 \mathrm{BL} / 6$ mice (64.3 versus 33.9\%). The time of onset of EAE was similar in C57BL/6 and IFNAR1-KO ( $13.4 \pm 0.6$ versus $14.8 \pm 0.7$ days). At onset of EAE, animals received anti-MOG $+\mathrm{C}$ or PBS by injection 
A

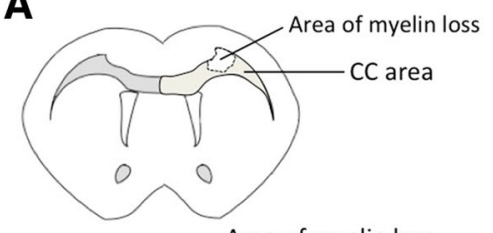

Demyelination $\%=\frac{\text { Area of myelin loss }}{\text { CC area }} * 100$

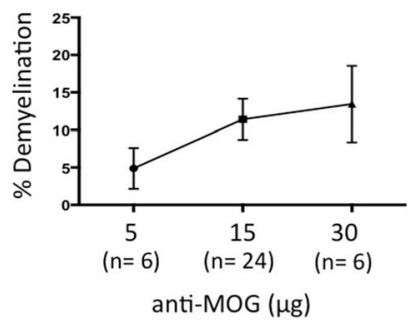

B
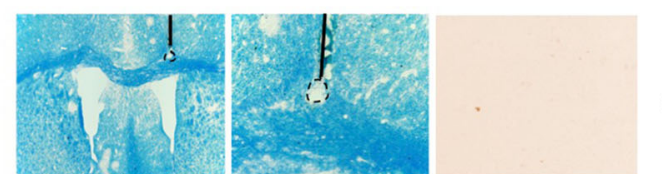

anti-MOG
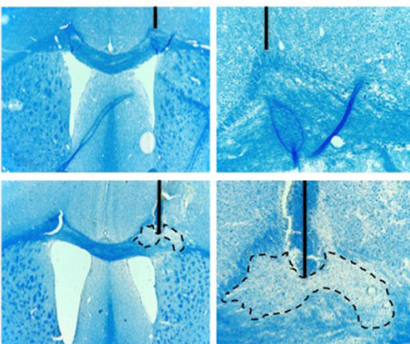

LFB

C9neo

C

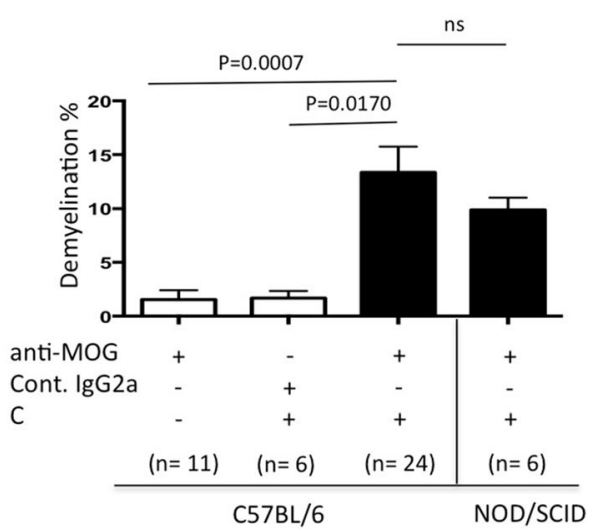

Fig. 1 Anti-MOG + C-mediated demyelination. a The left top panel shows a schematic drawing of the experimental setup showing how demyelination was quantified. The degree of demyelination in percentage was measured as the loss of myelin (area of myelin loss) divided by area of corpus callosum (CC area) in that hemisphere of the brain. The right top panel shows a dose response for demyelination in CC of C57BL/6 mice receiving purified monoclonal anti-MOG antibody from hybridoma clone Z2, along with C. Data are shown as mean \pm SEM, the total number of animals in each group is shown underneath columns, and this experiment was repeated only a single time with each dose. b Representative images of LFB and C9neo staining from C57BL/6 mice injected with anti-MOG, control IgG2a+C and anti-MOG + C. Magnification: LFB ( $\times 4$ and ×20) and C9neo ( $\times 20)$. c Quantitation of the area of myelin loss in CC in C57BL/6 mice 2 days after injection of anti-MOG, control lgG2a+C, and anti-MOG + C as well as NOD-Scid/J immunodeficient mice injected with anti-MOG $+C$ and pathology. Statistical significance was assessed using two-tailed Mann-Whitney $U$ test. Results are shown as mean \pm SEM, the total number of animals in each group is shown underneath columns. This experiment was repeated at least three times

to $\mathrm{CC}$ (Fig. 3a). As expected, EAE was characterized by prominent mononuclear cell infiltration throughout the leptomeninges and white matter in the spinal cord (Additional file 1). EAE severity at day 2 was $2.2 \pm 0.2$ and $3.2 \pm$ 0.6 for C57BL/6 mice injected with PBS and anti-MOG + $\mathrm{C}$, respectively, and $2.3 \pm 0.2$ and $3.0 \pm 0.4$ for IFNAR1-KO injected with PBS and anti-MOG $+C$, respectively. Importantly there was no detectable pathology in the $\mathrm{CC}$ in mice with EAE unless induced by focal injection of anti-
MOG and $C$ (Fig. 3b). Callosal demyelination induced by anti-MOG + C in C57BL/6 mice with pre-established EAE was similar to that in mice without disease. Strikingly, the anti-MOG + C-induced callosal demyelination was not different in IFNAR1-KO with pre-established EAE compared to $\mathrm{C} 57 \mathrm{BL} / 6$ mice.

The severity of EAE was not different between the groups treated with PBS or anti-MOG + C either in C57BL/6 or IFNAR1-KO, and the pattern of spinal cord 
A

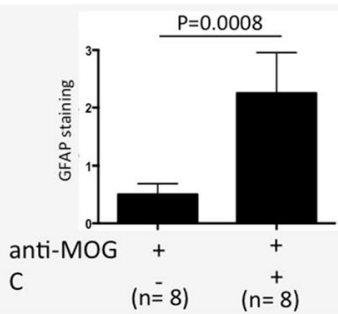

\section{Astrogliosis}

Anti-MOG $+\mathrm{C}$
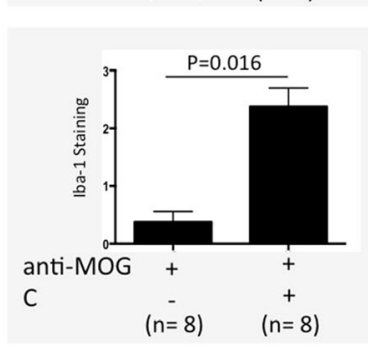

IFN- $\beta$

CXCL10
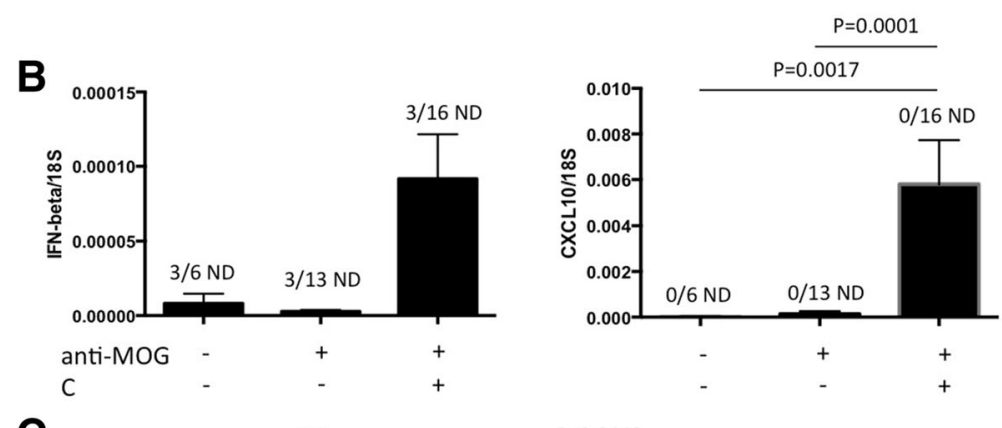

C

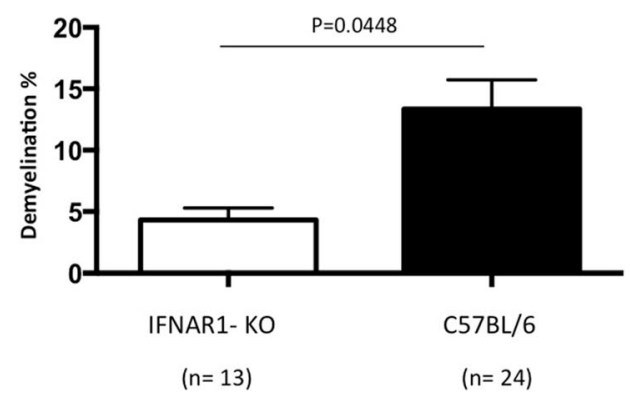

Fig. 2 The influence of type I IFN signaling on antibody-mediated demyelination. a The left panel shows a bar graph with activation of astrocytes and microglia in and around demyelinated areas in animals injected with anti-MOG $+\mathrm{C}$ or anti-MOG alone. The right panel shows representative images of GFAP and Iba-1 staining from mice injected with anti-MOG + C. Results in the bar graph are shown as mean \pm SEM of semiquantitative scores (arbitrary units), the total number of animals in each group is shown underneath columns. Original magnification in the representative images is $\times 20$. b Bar graphs showing IFN- $\beta$ and CXCL10 gene expression in animals injected with anti-MOG + C, PBS, or anti-MOG alone. Data are shown as mean \pm SEM, the total number of animals in each group is shown underneath columns. The data represent pooled results from at least three separate experiments. IFN- $\beta$ gene expression was not detected (ND) in three samples from each group. $\mathbf{c}$ Anti-MOG $+C$ was injected into CC of either C57BL/6 or IFNAR1-deficient mice, and pathology was assessed 2 days later. Bar graph shows percentage demyelination. Data were analyzed using two-tailed Mann-Whitney $U$ test. Results are shown as mean + SEM, the total number of animals in each group is shown underneath columns. The data represent pooled results from three separate experiments

infiltration in IFNAR1-KO and C57BL/6 mice was unaffected by intra-CC injection of anti-MOG $+\mathrm{C}$ or PBS (Additional file 1). To ask whether the equivalence of demyelination between WT and IFNAR1-KO mice with EAE could reflect inflammation-associated cytokines, we administered IFN- $\gamma$ to IFNAR1-KO mice. Figure 3c shows that co-injection of IFN- $\gamma$ into $\mathrm{CC}$ in IFNAR1-KO mice along with anti-MOG $+\mathrm{C}$ led to demyelination at levels equivalent to WT mice and so could compensate for the lack of type I IFN signaling.

\section{Discussion}

We have shown that a MOG-specific antibody induced $\mathrm{C}$-dependent demyelination in the $\mathrm{CC}$ of mice. 


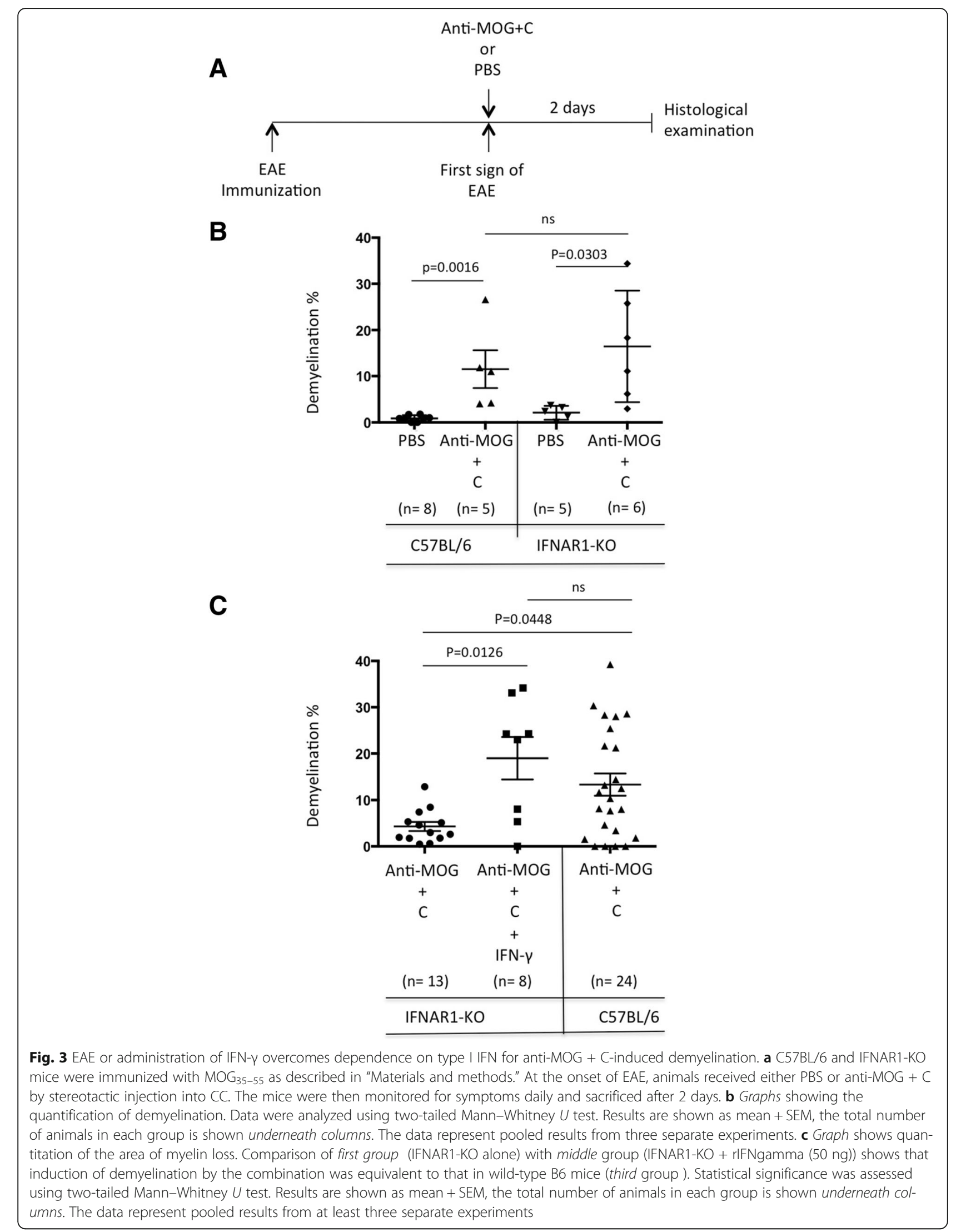


Demyelination was similarly induced in NOD-Scid/J immunodeficient mice that lack $\mathrm{T}$ and $\mathrm{B}$ cells. We observed that the type I IFN receptor is required for anti-MOG + $\mathrm{C}$-mediated demyelination. Remarkably, there was no effect of IFNAR1 deficiency on demyelination in CC in mice with pre-established EAE. Thus, induction of EAE overcame the dependence on type I IFN for anti-MOG $+\mathrm{C}$-mediated demyelination in the CC. We could also overcome the effect of IFNAR1 deficiency by coinjection of IFN- $\gamma$. These findings show sufficiency of myelin-specific antibody with $\mathrm{C}$ for white matter demyelination and a role for IFN signaling in promoting this. These findings are novel, representing direct demonstration of pathogenicity of monoclonal anti-MOG antibody in otherwise unmanipulated mice.

It has previously been shown that co-injection of antiMOG induced demyelination in an otherwise nondemyelinating uniphasic EAE model in Lewis rats [21]. Demyelination was observed in the perivascular cuffs and subpial bands of the white matter. Furthermore, high titer anti-MOG-containing guinea pig serum has been reported to induce demyelination when transferred into subarachnoid space of normal rats [22]. Demyelination that was observed in the spinal cord was presumed to reflect both the titer of antibody as well as serum complement. Our study has taken this approach further by using a monoclonal antibody of defined specificity in $\mathrm{CC}$ and examining the role of type I IFN.

In a previous study, we demonstrated type I IFN involvement in NMO-like pathology, showing reduced NMO-like pathology in mice lacking the receptor for type I IFN [24]. That finding was congruent with the clinical disease NMO, which is refractory to IFN- $\beta$ treatment and may even be exacerbated by it $[25,30]$. The involvement of type I IFN signaling in anti-MOG + $\mathrm{C}$-induced demyelination may reflect a general role for IFN signaling. The mechanism whereby IFN signaling promotes complement-dependent antibody-mediated pathology, clearly of importance for inflammatory demyelinating diseases such as MS, remains to be established.

By contrast with NMO, IFN- $\beta$ is used to treat MS and is effective against EAE. Over $50 \%$ of MS lesions have been shown to involve antibody and $\mathrm{C}$ deposition on demyelinating axons, so at first glance, it seems contradictory that IFN- $\beta$ should have therapeutic benefit for MS while promoting antibody-mediated demyelination. However, therapeutic application of IFN- $\beta$ in MS is via peripheral injection, and relapse prevention may depend on extra-CNS effects, as has been discussed [31]. Both type I and II IFNs are expressed in the CNS of MS patients [31, 32], so IFN-dependent pathology should not be impeded. Experimental models in which CNSendogenous type I IFN has been shown to be protective are not noted for their dependence on antibodymediated pathology [33] and indeed may not require antibodies or B cells at all [16]. Recently, it has been reported that treatment with IFN- $\beta$ in MOG-IgG-positive patients was associated with increasing relapse rate [14]. It can be speculated that the lack of benefit of IFN- $\beta$ in antibody-mediated diseases in CNS such as NMO may reflect the predominance of purely antibody-dependent pathology. Better definition of the relative roles of type I and II IFN in the CNS versus in the periphery will be required for fuller understanding of this complex interplay.

Stereotactic injection of a myelin-specific IgG antibody with $C$ to a white matter tract has allowed analysis of focal demyelinating lesions that are independent of $\mathrm{T}$ or $\mathrm{B}$ cells. This experimental system identifies sufficiency of antibody and C for MS-like pathology and a role for IFN signaling in promoting this. These findings will need to be integrated to the much more complex inflammatory environment in MS in which other pathological mechanisms and many cell types also contribute.

\section{Additional file}

\begin{abstract}
Additional file 1: H\&E and LFB staining of spinal cord sections from PBS- and anti-MOG + C-treated C57BL/6 and IFNAR1-KO mice with EAE. Shown is dorsal horn of the lumbar spinal cord taken 2 days post-intraCC injection. There was no significant difference in EAE severity between IFNAR1-KO and C57BL/6 mice. EAE severity was $2.2 \pm 0.2$ and $3.2 \pm 0.6$ for C57BL/6 mice injected with PBS and anti-MOG $+C$, respectively, and $2.3 \pm 0.2$ and $3.0 \pm 0.4$ for IFNAR1-KO injected with PBS and anti-MOG $+\mathrm{C}$, respectively. H\&E and LFB magnification: $\times 4$ and $\times 20$. (JPEG $375 \mathrm{~kb}$ )
\end{abstract}

\section{Abbreviations}

ADEM: Acute disseminated encephalomyelitis; AQP4: Aquaporin-4; C: Complement; CC: Corpus callosum; CNS: Central nervous system; CSF: Cerebrospinal fluid; EAE: Experimental autoimmune encephalomyelitis; H\&E: Hematoxylin and eosin; IFN: Interferon; IFNAR1-KO: Type I IFN receptor deficient mice; IFN- $\beta$ : IFN-beta; IFN- $\gamma$ : IFN-gamma; IgG: Immunoglobulin G; LFB: Luxol fast blue; MOG: Myelin oligodendrocyte glycoprotein; MS: Multiple sclerosis; NMO: Neuromyelitis optica; NMOSD: Neuromyelitis optica spectrum disorder; PBS: Phosphate-buffered saline; SEM: Standard error of mean

\section{Acknowledgements}

We thank Dina S. Arengoth and Pia Nyborg Nielsen for advice on animal handling and operating procedures and technical support, respectively. We thank Professor Chris Linington from University of Glasgow for providing us with the anti-MOG monoclonal antibody (Z2 hybridoma).

\section{Funding}

This research was supported by the Danish Multiple Sclerosis Society, Danish Agency for Science Technology and innovation, Fonden for lægevidenskabelige fremme, Civilingeniør Bent Bøgh og hustru Inge Bøghs Fond and Læge Else Poulsens Mindelegat.

Availability of data and materials

All raw data in this manuscript are available on request.

\section{Authors' contributions}

CTB was responsible for stereotactic injection and induction of EAE, animal care, keeping record of daily weights, neurological scoring of the EAE animals, manuscript preparation and performing RT-qPCR analysis, histology, and statistical analysis. TO, NA, and RK gave supervision on the design of the 
study and contributed significantly to the intellectual development of the project and helped to draft the manuscript. All authors critically revised the manuscript and approved the final manuscript.

\section{Competing interests}

The authors declare that they have no competing interests.

\section{Consent for publication}

Not applicable

\section{Ethics approval}

Animal experiments were approved by the Danish Animal Experiments Inspectorate (approval number 2014-15-0201-00369).

\section{Publisher's Note}

Springer Nature remains neutral with regard to jurisdictional claims in published maps and institutional affiliations.

\section{Author details}

${ }^{1}$ Institute of Molecular Medicine, Neurobiology, University of Southern Denmark, JB. Winsloewsvej 25, 5000 Odense C, Denmark. ²Department of Neurology, Slagelse Hospital, Institute of Regional Health Service Research, University of Southern Denmark, Odense, Denmark.

Received: 13 January 2017 Accepted: 13 June 2017

Published online: 24 June 2017

\section{References}

1. Compston A, Coles A. Multiple sclerosis. Lancet. 2008:372:1502-17.

2. Kutzelnigg A, Lucchinetti CF, Stadelmann C, Bruck W, Rauschka H, Bergmann M, Schmidbauer M, Parisi JE, Lassmann H. Cortical demyelination and diffuse white matter injury in multiple sclerosis. Brain. 2005;128:2705-12.

3. Lucchinetti C, Bruck W, Parisi J, Scheithauer B, Rodriguez M, Lassmann H. Heterogeneity of multiple sclerosis lesions: implications for the pathogenesis of demyelination. Ann Neurol. 2000;47:707-17.

4. Lucchinetti CF, Bruck W, Rodriguez M, Lassmann H. Distinct patterns of multiple sclerosis pathology indicates heterogeneity on pathogenesis. Brain Pathol. 1996;6:259-74.

5. Keegan M, Konig F, McClelland R, Bruck W, Morales Y, Bitsch A, Panitch H, Lassmann H, Weinshenker B, Rodriguez M, et al. Relation between humoral pathological changes in multiple sclerosis and response to therapeutic plasma exchange. Lancet. 2005;366:579-82.

6. Spadaro M, Gerdes LA, Mayer MC, Ertl-Wagner B, Laurent S, Krumbholz M, Breithaupt C, Hogen T, Straube A, Giese A, et al. Histopathology and clinical course of MOG-antibody-associated encephalomyelitis. Ann Clin Transl Neurol. 2015:2:295-301.

7. Jarius S, Metz I, Konig FB, Ruprecht K, Reindl M, Paul F, Bruck W, Wildemann B. Screening for MOG-lgG and 27 other anti-glial and antineuronal autoantibodies in 'pattern II multiple sclerosis' and brain biopsy findings in a MOG-IgG-positive case. Mult Scler. 2016;22:1541-9.

8. Di Pauli F, Hoftberger R, Reindl M, Beer R, Rhomberg P, Schanda K, Sato D, Fujihara K, Lassmann $\mathrm{H}$, Schmutzhard $\mathrm{E}$, Berger T. Fulminant demyelinating encephalomyelitis: Insights from antibody studies and neuropathology. Neurol Neuroimmunol Neuroinflamm. 2015;2, e175

9. Brunner C, Lassmann H, Waehneldt TV, Matthieu JM, Linington C. Differential ultrastructural localization of myelin basic protein, myelin/oligodendroglial glycoprotein, and 2',3'-cyclic nucleotide 3'-phosphodiesterase in the CNS of adult rats. J Neurochem. 1989;52:296-304.

10. Lalive PH, Molnarfi N, Benkhoucha M, Weber MS, Santiago-Raber ML. Antibody response in MOG(35-55) induced EAE. J Neuroimmunol. 2011;240-241:28-33.

11. McLaughlin KA, Chitnis T, Newcombe J, Franz B, Kennedy J, McArdel S, Kuhle J, Kappos L, Rostasy K, Pohl D, et al. Age-dependent B cell autoimmunity to a myelin surface antigen in pediatric multiple sclerosis. J Immunol. 2009;183:4067-76

12. Kim SM, Woodhall MR, Kim JS, Kim SJ, Park KS, Vincent A, Lee KW, Waters P. Antibodies to MOG in adults with inflammatory demyelinating disease of the CNS. Neurol Neuroimmunol Neuroinflamm. 2015;2, e163.

13. Jarius S, Kleiter I, Ruprecht K, Asgari N, Pitarokoili K, Borisow N, Hummert MW, Trebst C, Pache F, Winkelmann A, et al. MOG-lgG in NMO and related disorders: a multicenter study of 50 patients. Part 3:
Brainstem involvement - frequency, presentation and outcome. J Neuroinflammation. 2016:13:281.

14. Jarius S, Ruprecht K, Kleiter I, Borisow N, Asgari N, Pitarokoili K, Pache F, Stich O, Beume LA, Hummert MW, et al. MOG-IgG in NMO and related disorders: a multicenter study of 50 patients. Part 2: Epidemiology, clinical presentation, radiological and laboratory features, treatment responses, and long-term outcome. J Neuroinflammation. 2016;13:280.

15. Pache F, Zimmermann H, Mikolajczak J, Schumacher S, Lacheta A, Oertel FC, Bellmann-Strobl J, Jarius S, Wildemann B, Reindl M, et al. MOG-lgG in NMO and related disorders: a multicenter study of 50 patients. Part 4: Afferent visual system damage after optic neuritis in MOG-lgG-seropositive versus AQP4-IgG-seropositive patients. J Neuroinflammation. 2016;13:282

16. Lyons JA, San M, Happ MP, Cross AH. B cells are critical to induction of experimental allergic encephalomyelitis by protein but not by a short encephalitogenic peptide. Eur J Immunol. 1999;29:3432-9.

17. Kuerten S, Pauly R, Rottlaender A, Rodi M, Gruppe TL, Addicks K, Tary-Lehmann M, Lehmann PV. Myelin-reactive antibodies mediate the pathology of MBP-PLP fusion protein MP4-induced EAE. Clin Immunol. 2011:140:54-62.

18. Lyons JA, Ramsbottom MJ, Cross AH. Critical role of antigen-specific antibody in experimental autoimmune encephalomyelitis induced by recombinant myelin oligodendrocyte glycoprotein. Eur J Immunol. 2002;32:1905-13.

19. Molnarfi N, Schulze-Topphoff U, Weber MS, Patarroyo JC, Prod'homme T, Varrin-Doyer M, Shetty A, Linington C, Slavin AJ, Hidalgo J, et al. MHC class II-dependent B cell APC function is required for induction of CNS autoimmunity independent of myelin-specific antibodies. J Exp Med. 2013;210:2921-37.

20. Oliver AR, Lyon GM, Ruddle NH. Rat and human myelin oligodendrocyte glycoproteins induce experimental autoimmune encephalomyelitis by different mechanisms in C57BL/6 mice. J Immunol. 2003:171:462-8.

21. Schluesener HJ, Sobel RA, Linington C, Weiner HL. A monoclonal antibody against a myelin oligodendrocyte glycoprotein induces relapses and demyelination in central nervous system autoimmune disease. J Immunol. 1987:139:4016-21.

22. Linington $\mathrm{C}$, Lassmann $\mathrm{H}$. Antibody responses in chronic relapsing experimental allergic encephalomyelitis: correlation of serum demyelinating activity with antibody titre to the myelin/oligodendrocyte glycoprotein (MOG). J Neuroimmunol. 1987:17:61-9.

23. Asgari N, Khorooshi R, Lillevang ST, Owens T. Complement-dependent pathogenicity of brain-specific antibodies in cerebrospinal fluid. J Neuroimmunol. 2013:254:76-82

24. Khorooshi R, Wlodarczyk A, Asgari N, Owens T. Neuromyelitis optica-like pathology is dependent on type I interferon response. Exp Neurol. 2013;247:744-7

25. Palace J, Leite MI, Nairne A, Vincent A. Interferon Beta treatment in neuromyelitis optica: increase in relapses and aquaporin 4 antibody titers. Arch Neurol. 2010;67:1016-7.

26. Kim SH, Kim W, Li XF, Jung IJ, Kim HJ. Does interferon beta treatment exacerbate neuromyelitis optica spectrum disorder? Mult Scler. 2012:18:1480-3.

27. Tanaka M, Tanaka K, Komori M. Interferon-beta(1 b) treatment in neuromyelitis optica. Eur Neurol. 2009:62:167-70.

28. Asgari N, Kyvik KO, Steenstrup T, Stenager E, Lillevang ST. Antibodies against interferon-beta in neuromyelitis optica patients. J Neurol Sci. 2014:339:52-6.

29. Wlodarczyk A, Cedile O, Jensen KN, Jasson A, Mony JT, Khorooshi R, Owens T. Pathologic and Protective Roles for Microglial Subsets and Bone Marrow- and Blood-Derived Myeloid Cells in Central Nervous System Inflammation. Front Immunol. 2015:6:463.

30. Asgari N, Voss A, Steenstrup T, Kyvik KO, Stenager E, Lillevang ST. Interferon alpha association with neuromyelitis optica. Clin Dev Immunol. 2013:2013: 713519.

31. Owens T, Khorooshi R, Wlodarczyk A, Asgari N. Interferons in the central nervous system: a few instruments play many tunes. Glia. 2014:62:339-55.

32. Goverman J. Autoimmune T cell responses in the central nervous system. Nat Rev Immunol. 2009;9:393-407.

33. Khorooshi R, Morch MT, Holm TH, Berg CT, Dieu RT, Draeby D, Issazadeh-Navikas S, Weiss S, Lienenklaus S, Owens T. Induction of endogenous Type I interferon within the central nervous system plays a protective role in experimental autoimmune encephalomyelitis. Acta Neuropathol. 2015;130:107-18. 